\title{
Isolates from hospital environments are the most virulent of the Candida parapsilosis complex
}

Raquel Sabino ${ }^{1,2+}$, Paula Sampaio ${ }^{1 \dagger}$, Catarina Carneiro ${ }^{1}$, Laura Rosado ${ }^{2}$ and Célia Pais ${ }^{1 *}$

\begin{abstract}
Background: Candida parapsilosis is frequently isolated from hospital environments, like air and surfaces, and causes serious nosocomial infections. Molecular studies provided evidence of great genetic diversity within the $C$. parapsilosis species complex but, despite their growing importance as pathogens, little is known about their potential to cause disease, particularly their interactions with phagocytes. In this study, clinical and environmental C. parapsilosis isolates, and strains of the related species C. orthopsilosis and C. metapsilosis were assayed for their ability to induce macrophage cytotocixity and secretion of the pro-inflammatory cytokine TNF- $\alpha$, to produce pseudo-hyphae and to secrete hydrolytic enzymes.

Results: Environmental C. parapsilosis isolates caused a statistically significant ( $p=0.0002$ ) higher cell damage compared with the clinical strains, while C. orthopsilosis and C. metapsilosis were less cytotoxic. On the other hand, clinical isolates induced a higher TNF- $\alpha$ production compared with environmental strains $(p<0.0001)$. Whereas the amount of TNF- $\alpha$ produced in response to C. orthopsilosis strains was similar to the obtained with C. parapsilosis environmental isolates, it was lower for C. metapsilosis strains. No correlation between pseudo-hyphae formation or proteolytic enzymes secretion and macrophage death was detected $(p>0.05)$. However, a positive correlation between pseudo-hyphae formation and TNF- $\alpha$ secretion was observed ( $p=0.0119)$.

Conclusions: We show that environmental C. parapsilosis strains are more resistant to phagocytic host defences than bloodstream isolates, being potentially more deleterious in the course of infection than strains from a clinical source. Thus, active environmental surveillance and application of strict cleaning procedures should be implemented in order to prevent cross-infection and hospital outbreaks.
\end{abstract}

\section{Background}

Candida parapsilosis is a human commensal of epithelial and mucosal tissues, also frequently isolated from hospital environments, like air and surfaces. It is the cause of serious nosocomial infections, being the second most common fungal species isolated from blood in many regions of the world [1-3]. Due to its association with parenteral nutrition and intravascular catheters, $C$. parapsilosis affects mainly critically ill patients from surgical intensive care units, neonates, and cancer patients [4-6]. Neonates are especially prone to candidemia, and in low weight infants the estimated incidence of invasive

\footnotetext{
*Correspondence: cpais@bio.uminho.pt

+ Contributed equally

'CBMA - Centre of Molecular and Environmental Biology, Department of

Biology, University of Minho, Braga, Portugal

Full list of author information is available at the end of the article
}

infections due to C. parapsilosis is $2 \%$, reaching as much as $10 \%$ in extreme cases [7-9].

The modes of transmission and portals of entry of fungal nosocomial infections vary according to the pathogen involved. Candida infections are predominantly of endogenous origin but cross-infection via hands of health care workers or relatives, or through devices has been shown to occur [10]. Invasive fungal infections may be acquired in the hospital from different sources, and numerous fungal reservoirs have been identified in hospital environment, including unfiltered air, ventilation systems, contaminated dust during hospital construction, carpeting, water, food, and ornamental plants [11]. In fact, environmental exposure to C. parapsilosis from hospital healthcare workers has been associated with both sporadic cases and outbreaks of invasive fungal infections in immunocompromised patients $[12,13]$.
C Biomed Central

() 2011 Sabino et al; licensee BioMed Central Ltd. This is an Open Access article distributed under the terms of the Creative Commons Attribution License (http://creativecommons.org/licenses/by/2.0), which permits unrestricted use, distribution, and reproduction in any medium, provided the original work is properly cited. 
Most pathogenic Candida species have developed a wide range of putative virulence factors to assist in their ability to colonize host tissues, cause disease, and overcome host defenses. Among them, secretion of hydrolytic enzymes such as aspartic proteinases and lipases, as well as morphogenesis have been well studied in C. albicans [14-16]. However, despite the growing importance of the C. parapsilosis species complex, few works evaluating the in vitro virulence of these species have been performed [17-19] and little is known about the virulence traits that enable them to cause disease.

Mononuclear phagocytes play an important role in innate immunity, in the polarization of the immune adaptive response and also in the eradication of Candida sp. [20,21]. Given the critical role played by macrophages in balancing colonization/infection, the analysis of their interaction with isolates belonging to the $C$. parapsilosis complex is important to understand the virulence potential of these species.

In the present work, we compared C. parapsilosis bloodstream isolates and strains recovered from the hospital setting regarding their virulence in vitro. Mononuclear phagocytes were used to test the strain ability to: (i) induce cytotocixity; (ii) activate TNF- $\alpha$ release; (iii) filament in vitro, both during macrophage infection and in the presence of serum, and (iv) secrete hydrolytic enzymes. Candida parapsilosis environmental isolates revealed to be the most virulent to macrophage cells, being potentially more deleterious, particularly in the initial phases of the infection, than strains from a clinical source.

\section{Results}

\section{Candida parapsilosis interaction with macrophages}

The ability of macrophages to kill C. parapsilosis bloodstream isolates and environmental strains was determined by CFU counting after one hour co-incubation, using six isolates of each. The average percentage of yeast killing for the environmental isolates was $10.97 \pm 2.67$ while for clinical isolates it was $33.22 \pm 5.25$, the difference being statistically significant $(p=0.0409)$. The interaction of one clinical and one environmental isolate with macrophages was followed for 12 hours of incubation. Microscopic examination showed that the clinical isolate was able to produce pseudo-hyphae and maintained that ability in contact with macrophages (Figure 1a and 1b), while the environmental isolate kept the yeast unicellular morphology (Figure 1c to 1e).

The percentage of dead macrophages after co-incubation with the same two isolates, assessed by propidium iodide (PI) staining, showed that macrophage killing did not vary significantly in the first 8 hours of incubation, with percentages of macrophage death similar to the negative control (Figure 2 and 3). However, after 12 hours of infection with the clinical isolate the percentage of macrophage killing increased to 41\% (Figure 2c, 12

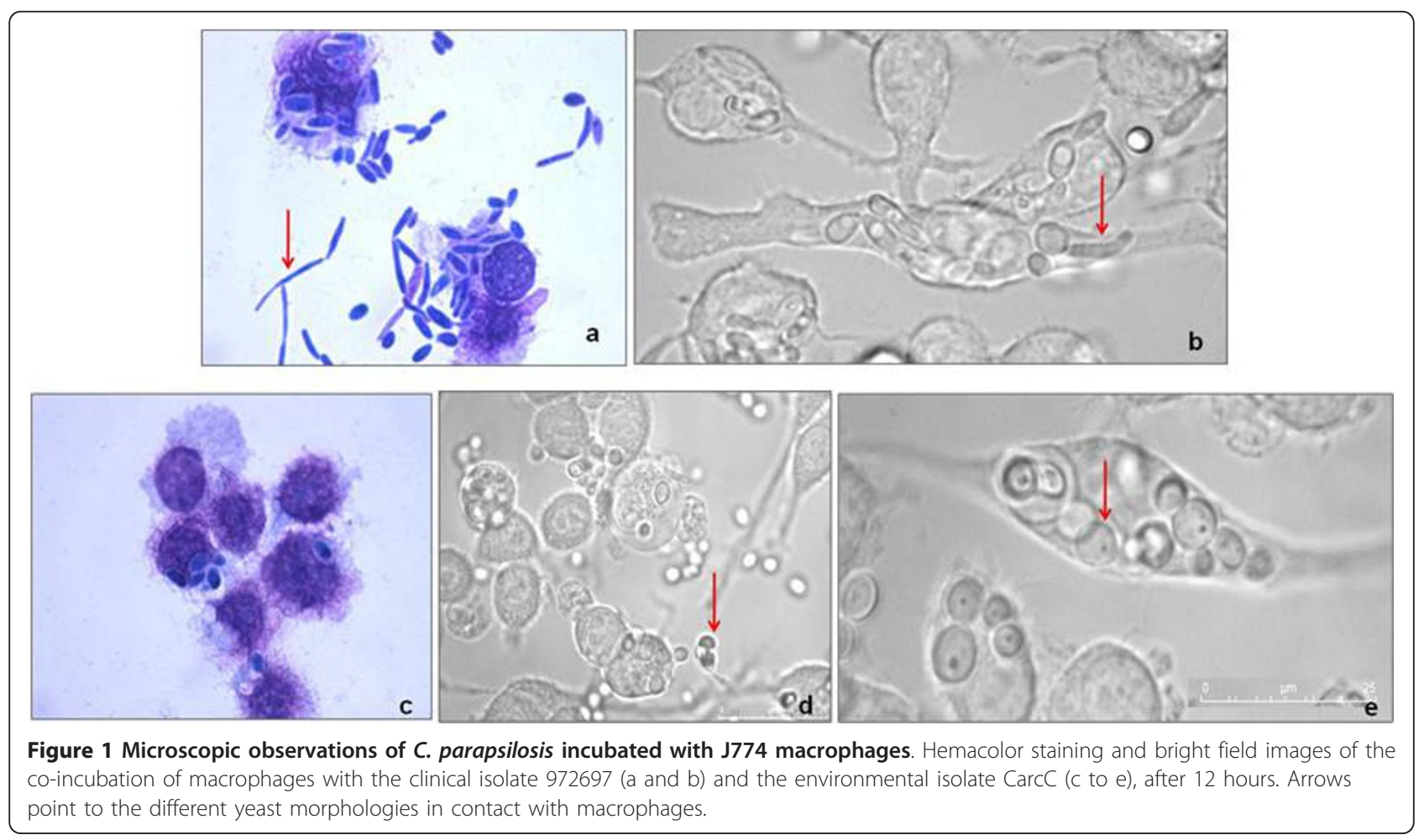




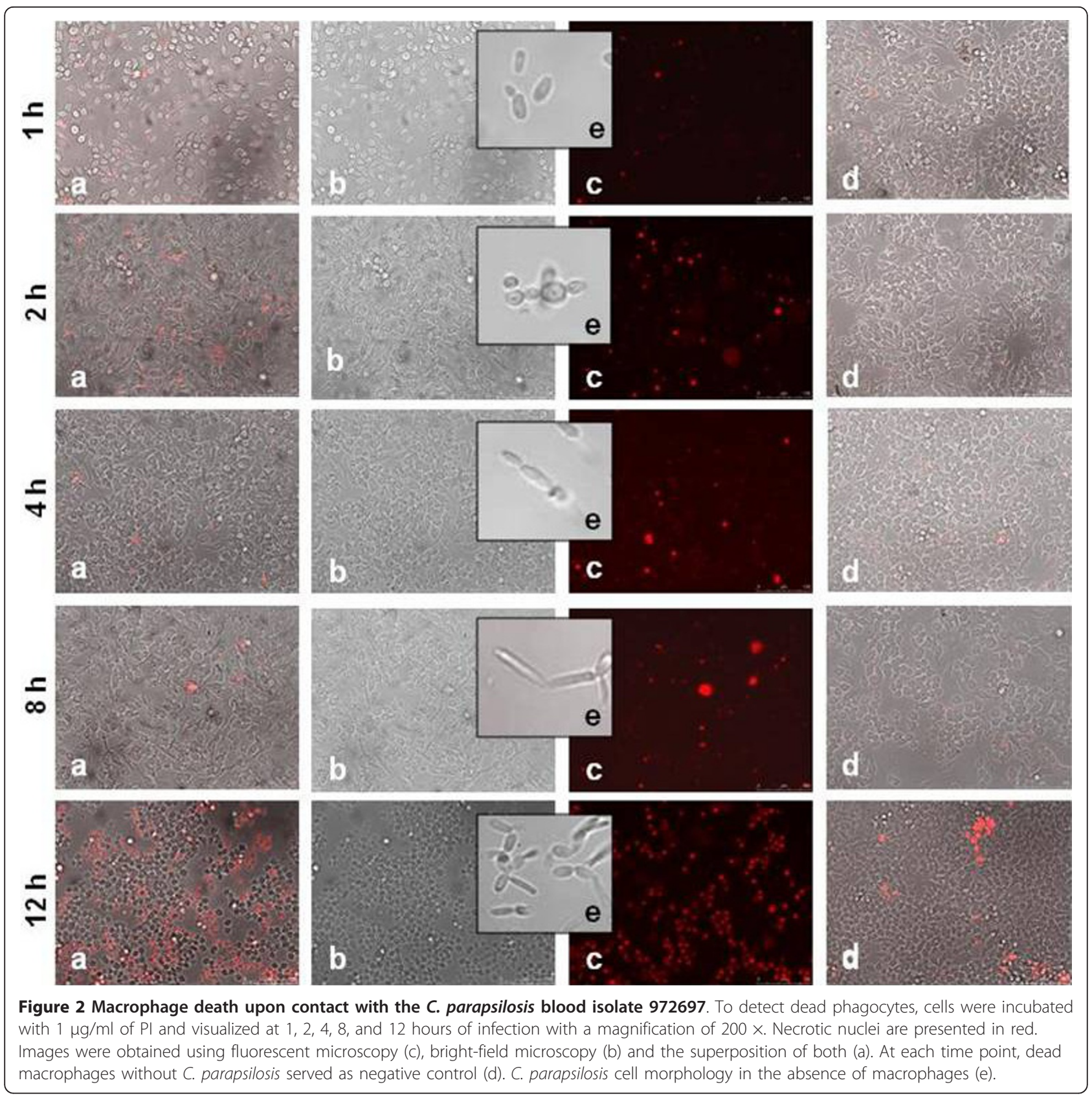

h). On the contrary, after 12 hours co-incubation with the environmental strain, the number of macrophages in the slide was significantly reduced (Figure 3a, b, $12 \mathrm{~h}$ ) when compared with the first hours of infection, and with the negative control (Figure 3d, $12 \mathrm{~h}$ ) and many yeast cells could be observed. Therefore, in this case, the proportion of PI positive cells could not be quantified due to the reduction of macrophage cell numbers, probably by cell lysis. Together, these observations suggested that clinical and environmental isolates behave differently in contact with macrophages. Although unable to filament, the environmental isolate induced macrophage cytotoxicity and cell lysis, which is consistent with the lower yeast killing observed previously. Following these results, twenty-five blood isolates and twenty environmental isolates were selected to test these findings, and the studies were extended to eight $C$. orthopsilosis and four C. metapsilosis strains, for comparison.

\section{Candida parapsilosis environmental isolates are more cytotoxic to macrophages}

The release of LDH by macrophages was monitored after 12 hours of co-incubation using all the different strains analysed in this study (Table 1). Results showed 


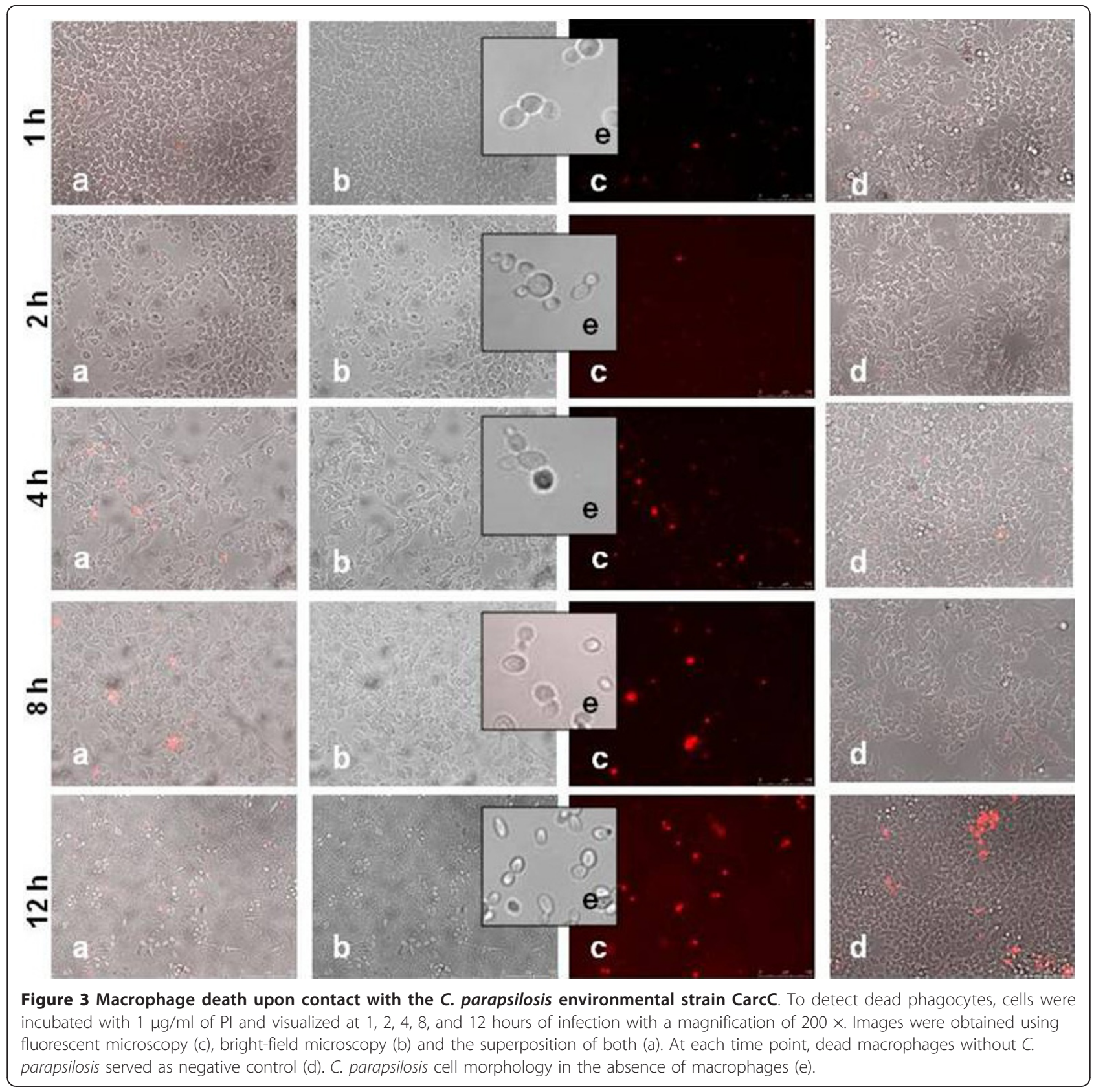

that the percentage of cytotoxicity varied from $6.4 \%$ to $59.2 \%$, revealing a great variability in strain ability to induce damage. Due to this variability the isolates were grouped into two classes of cytotoxicity and it was observed that the great majority of environmental strains exhibited cytotoxicity levels between 30.1 and $60.0 \%$, while clinical isolates were mainly in the group presenting 1 to $30 \%$ cytotoxicity (Figure 4 ). Overall, the environmental isolates induced statistically significant $(p$ $<0.0001$ ) higher cell damage (average 37.6\% \pm 13.78 ) when compared with the clinical strains $(22.9 \pm 10.36)$. Regarding C. orthopsilosis and C. metapsilosis the average percentage of induced cytotoxicity was $19.3 \%( \pm$ $6.17)$ and $8.8 \%( \pm 1.05)$, respectively.

Clinical isolates of $C$. parapsilosis are able to induce a higher inflammatory response in infected macrophages The amount of TNF- $\alpha$ released by infected macrophages was quantified as an indication of the yeast potential to induce an inflammatory response. TNF- $\alpha$ released varied from 50.51 to $809.4 \mathrm{pg} / \mathrm{ml}$ (Figure 5). The blood isolates induced a higher TNF- $\alpha$ secretion (average $557.7 \pm 190.95 \mathrm{pg} / \mathrm{ml}$ ) compared with the environmental strains (average $234.6 \pm 108.7 \mathrm{pg} / \mathrm{ml}$ ) and 
Table 1 Species used in this study, their collection date, and origin

\begin{tabular}{|c|c|c|c|c|c|}
\hline & Species & Isolate identification & Geographical origin & Collection date & Product \\
\hline \multirow[t]{20}{*}{ Environmental } & C. parapsilosis & IPOA1 & Portugal - Hospital 1 & 2007 & Water tap nursery 23 \\
\hline & C. parapsilosis & IPOA2 & Portugal - Hospital 1 & 2007 & Bedside table no. 4 nursery 30 \\
\hline & C. parapsilosis & IPOA3 & Portugal - Hospital 1 & 2007 & Water tap nursery 24 \\
\hline & C. parapsilosis & IPOA14 & Portugal - Hospital 1 & 2007 & Treatment room \\
\hline & C. parapsilosis & IPOA15 & Portugal - Hospital 1 & 2007 & Door knob Patients' WC \\
\hline & C. parapsilosis & IPOA20 & Portugal - Hospital 1 & 2007 & Air from individual room no. 5 \\
\hline & C. parapsilosis & IPOA21 & Portugal - Hospital 1 & 2007 & Water tap treatment room \\
\hline & C. parapsilosis & IPOA22 & Portugal - Hospital 1 & 2007 & Shower Patients' WC \\
\hline & C. parapsilosis & IPOA23 & Portugal - Hospital 1 & 2007 & Air from nursery 24 \\
\hline & C. parapsilosis & CNR40 & France & 2007 & Hospital environment \\
\hline & C. parapsilosis & $494 \mathrm{~F}$ & France & 2007 & Hospital environment \\
\hline & C. parapsilosis & Carc & Portugal & 2006 & Beach sand \\
\hline & C. parapsilosis & Avc & Portugal & 2006 & Beach sand \\
\hline & C. parapsilosis & Pr b & Portugal & 2006 & Beach sand \\
\hline & C. parapsilosis & 1144 & Portugal - Hospital 2 & 2006 & Hospital air \\
\hline & C. parapsilosis & 1156 & Portugal - Hospital 2 & 2006 & Hospital air \\
\hline & C. parapsilosis & 1159 & Portugal - Hospital 2 & 2006 & Hospital air \\
\hline & C. parapsilosis & 1160 & Portugal - Hospital 2 & 2006 & Hospital air \\
\hline & C. parapsilosis & 1182 & Portugal - Hospital 2 & 2006 & Hospital air \\
\hline & C. parapsilosis & 1194 & Portugal - Hospital 2 & 2006 & Hospital air \\
\hline \multirow[t]{31}{*}{ Clinical } & C. parapsilosis & 376604 & Portugal - Hospital 1 & 2002 & Blood culture \\
\hline & C. parapsilosis & 378058 & Portugal - Hospital 1 & 2002 & Blood culture \\
\hline & C. parapsilosis & 378690 & Portugal - Hospital 1 & 2002 & Blood culture \\
\hline & C. parapsilosis & 433573 & Portugal - Hospital 1 & 2003 & Blood culture \\
\hline & C. parapsilosis & 431472 & Portugal - Hospital 1 & 2003 & Blood culture \\
\hline & C. parapsilosis & 476446 & Portugal - Hospital 1 & 2003 & Blood culture \\
\hline & C. parapsilosis & 506858 & Portugal - Hospital 1 & 2003 & Blood culture \\
\hline & C. parapsilosis & 522760 & Portugal - Hospital 1 & 2004 & Blood culture \\
\hline & C. parapsilosis & 864647 & Portugal - Hospital 1 & 2006 & Blood culture \\
\hline & C. parapsilosis & 814455 & Portugal - Hospital 1 & 2006 & Blood culture \\
\hline & C. parapsilosis & 972697 & Portugal - Hospital 1 & 2007 & Blood culture \\
\hline & C. parapsilosis & $20 \mathrm{~L}$ & France & 2004 & Blood culture \\
\hline & C. parapsilosis & 155 & France & 2004 & Blood culture \\
\hline & C. parapsilosis & 202 & France & 2004 & Blood culture \\
\hline & C. parapsilosis & 272 & France & 2004 & Blood culture \\
\hline & C. parapsilosis & 465 & France & 2005 & Blood culture \\
\hline & C. parapsilosis & 573 & France & 2005 & Blood culture \\
\hline & C. parapsilosis & 648 & France & 2006 & Blood culture \\
\hline & C. parapsilosis & 899 & France & 2006 & Blood culture \\
\hline & C. parapsilosis & CAN16 & Portugal - Hospital 3 & 2002 & Blood culture \\
\hline & C. parapsilosis & CAN159 & Portugal - Hospital 3 & 2004 & Blood culture \\
\hline & C. parapsilosis & CAN201 & Portugal - Hospital 3 & 2005 & Blood culture \\
\hline & C. parapsilosis & CAN270 & Portugal - Hospital 3 & 2006 & Blood culture \\
\hline & C. parapsilosis & CAN279 & Portugal - Hospital 3 & 2007 & Blood culture \\
\hline & C. parapsilosis & $\mathrm{H} 1$ & USA & - & Blood culture \\
\hline & C. ortopsilosis & 754 & Portugal - Hospital 2 & 2004 & Bronchial secretions \\
\hline & C. ortopsilosis & 755 & Portugal - Hospital 2 & 2004 & Bronchial secretions \\
\hline & C. ortopsilosis & 892 & Portugal - Hospital 2 & 2004 & Blood culture \\
\hline & C. ortopsilosis & 894 & Portugal - Hospital 2 & 2004 & Blood culture \\
\hline & C. ortopsilosis & 895 & Portugal - Hospital 2 & 2004 & Blood culture \\
\hline & C. ortopsilosis & 981224 & USA & - & Unknown \\
\hline
\end{tabular}


Table 1 Species used in this study, their collection date, and origin (Continued)

\begin{tabular}{lcccc}
\hline C. ortopsilosis & H10 & USA & - & Unknown \\
C. ortopsilosis & CAN 138 & Portugal - Hospital 3 & 2004 & Blood culture \\
C. metapsilosis & 911012 & Portugal - Hospital 1 & 2006 & Blood culture \\
C. metapsilosis & CAN 155 & Portugal - Hospital 3 & 2004 & Blood culture \\
C. metapsilosis & 960161 & USA & - & Unknown \\
C. metapsilosis & am 2006 & USA & - & Unknown \\
\hline
\end{tabular}

this difference was statistically significant $(p<0.0001)$. The average amount of TNF- $\alpha$ production by $C$. orthopsilosis strains was $204.6 \pm 77.40 \mathrm{pg} / \mathrm{ml}$, similar to $C$. parapsilosis environmental isolates, whereas for $C$. metapsilosis only $75.4 \pm 23.84 \mathrm{pg} / \mathrm{ml}$ was detected. All comparisons were statistically significant $(p<0.05)$ except for C. orthopsilosis vs environmental C. parapsilosis strains.

\section{Pseudo-hyphae formation and secretion of aspartic proteinase and phospholipase}

Virulence factors such as secretion of hydrolytic enzymes, aspartic proteinases and/or phospholipases, and pseudo-hyphae formation are likely to contribute to Candida cytotoxicity. These characteristics were measured in all isolates used in this study and results are shown in Table 2 . About $60 \%$ of $C$. parapsilosis isolates were able to produce pseudo-hyphae after 12 hours of incubation. Interestingly, comparing environmental with clinical isolates, the majority of the pseudo-hyphae producers were the clinical ones, and this difference was statistically significant $\left(\chi^{2}=4.664, p=0.0154\right)$. Around half of the $C$. orthopsilosis strains produced pseudohyphae, while none of the C. metapsilosis isolates was able to filament.

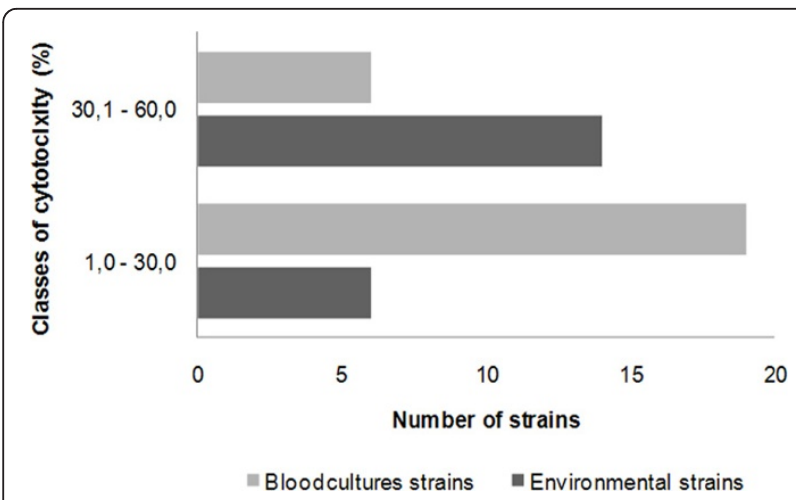

Figure 4 Distribution of $C$. parapsilosis environmental and blood isolates, according to the defined classes of cytotoxicity. The majority of environmental isolates are included in the group causing between 30 and 60\% cytotoxicity. Cellular damage induced by the yeast was quantified as the amount of $\mathrm{LDH}$ release by macrophages after 12 hours of infection.
High proteinase activity was found in 36 (80.0\%) C. parapsilosis strains, being $38.8 \%$ environmental and $61.2 \%$ clinical isolates (Table 2). However, no significant difference $\left(\chi^{2}=2.250, p=0.0688\right)$ was observed when comparing environmental and clinical isolates. No Sap production was observed in most of the C. orthopsilosis and $C$. metapsilosis isolates (Table 2). No significant phospholipase production was detected in the tested isolates.

\section{Discussion}

Candida parapsilosis accounts for a significant proportion of nosocomial infections, with an increasing prevalence in hospital settings. As with other Candida species, invasion of $C$. parapsilosis can result in severe disease, particularly in hosts with a compromised immune system. Unlike $C$. albicans, the transmission and acquisition of infection due to $C$. parapsilosis is mainly exogenous and environmental strains are often the source of infection. The main issue of this study was, therefore, the comparison of the virulence potential of environmental and clinical C. parapsilosis isolates.

Macrophages play an important role in the immune response, directly by phagocytosing and killing microbial pathogens, and indirectly by processing and presenting antigens and secreting cytokines [22]. Although there were variations in the intracellular killing of the different strains, the average percentage was of about $35 \%$ for the clinical isolates, in agreement with the results obtained by Gácser et al. [18] for C. parapsilosis. Curiously, these

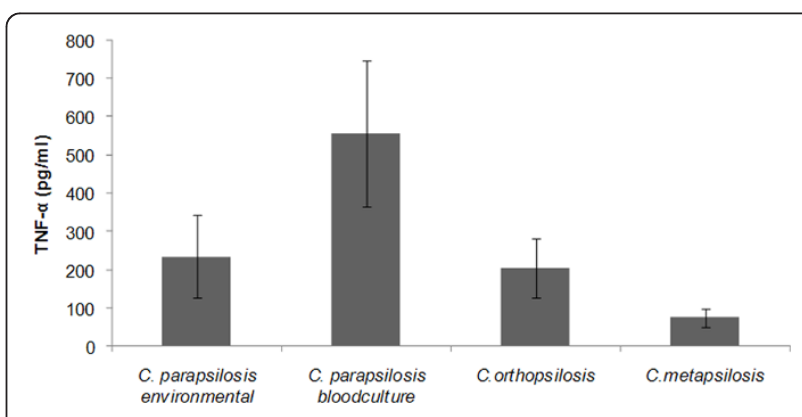

Figure 5 Determination of TNF- $\alpha$ release. Level of TNF- $\alpha$ release by macrophages infected with environmental and bloodculture $C$. parapsilosis isolates, and with C. orthopsilosis, and C. metapsilosis isolates after 12 hours of infection. 
Table 2 Pseudo-hyphae and secreted aspartyl proteinase (sap) production

\begin{tabular}{lllll}
\hline Isolates & \multicolumn{2}{l}{ Pseudo-hyphae production } & \multicolumn{2}{c}{ Sap production } \\
\cline { 2 - 5 } & Yes & No & High & Low \\
\hline C. parapsilosis & & 12 & 14 & 6 \\
Environment & 8 & 7 & 22 & 3 \\
Bloodcultures & 18 & 5 & 2 & 6 \\
C. orthopsilosis & 3 & 4 & 0 & 4 \\
C. metapsilosis & 0 & 28 & 38 & 19 \\
Total no. of strains & 29 &
\end{tabular}

Study of 45 environmental and clinical Candida parapsilosis isolates, $8 \mathrm{C}$. orthopsilosis and 4 C. metapsilosis strains.

values were much lower for the environmental strains, pointing to a clear difference between environmental and clinical isolates, regarding interaction with macrophages. A great variability in the capacity of the strains to cause cell damage was also found, and again environmental isolates induced significantly higher macrophage damage than blood isolates, confirming a strong relationship between the source of the isolates and their ability to cause damage. It was also observed that $C$. orthopsilosis induced a high level of macrophage damage, similar to $C$. parapsilosis bloodstream isolates, while $C$. metapsilosis induced the lowest cytotoxicity level. These facts agree with previous works on reconstituted human oral epithelial and epidermal tissues [19] and microglial cells [23], showing that $C$. metapsilosis was less virulent compared to C. orthopsilosis and C. parapsilosis. To correlate these findings with the morphology, yeast strains were induced to filament in the presence of serum and results showed that $57.7 \%$ of the tested C. parapsilosis isolates were able to produce pseudo-hyphae after 12 hours of incubation, with the clinical isolates filamenting in a higher percentage than the environmental strains. Curiously, this high filamentation ability was not correlated with higher macrophage cytotoxicity as it has been described for $C$. albicans $[24,25]$. In our study, although C. parapsilosis filamentation occurred right after 4 hours, differences in macrophage death were observed only after 12 hours of co-incubation. Incubation with the strains that did not develop pseudo-hyphae revealed that, after 12 hours of infection, a huge number of macrophages had disappeared and the yeast number was high. This observation could be explained by the hypothesis that yeast replicate inside macrophages and lyse them, enabling the release of yeast into the medium, as well as LDH. Since filamentation was not responsible for the death of the macrophages incubated with the environmental strains, maybe other virulence factors could account for these observations.

Secretion of hydrolytic enzymes such as aspartic proteinases and phospholipases have been associated with
C.albicans virulence $[14,16,26,27]$ and also with C. parapsilosis virulence $[15,18,28-31]$. Eighty percent of the tested C. parapsilosis strains were found to have high proteinase activity, being the majority blood isolates. To our knowledge, no other study compared Sap production in clinical and environmental C. parapsilosis isolates, but Dagdeviren et al. [32] observed a higher production of acid proteinase among C. parapsilosis blood isolates compared to non-blood isolates. From the eight C. orthopsilosis tested only $25 \%$ were Sap producers, whereas none of the C. metapsilosis was. This is in accordance with Lin et al. [33], who also reported differences in proteinase activity within the three major groups of C. parapsilosis.

No correlation was observed between hydrolytic enzymes secretion and environmental or clinical isolates, or with cell damage ( $\mathrm{p}>0.05$ ).

Macrophage activation induces releasing of several key mediators, including proinflammatory cytokines such as TNF- $\alpha$, which are important for protecting the host against disseminated candidiasis [34-36]. The amount of TNF- $\alpha$ produced by macrophages infected with C. parapsilosis isolates from bloodcultures was significantly higher than the amount produced by macrophages infected with environmental isolates, indicating that clinical isolates induce a higher pro-inflammatory response than environmental strains. The fact that a high macrophage cell lysis occurred in the co-incubations with the environmental strains could also account for these results. In contrast, Orsi et al. [23] reported little or no TNF- $\alpha$ production in the co-incubations of strains of the C. parapsilosis complex with microglial cells. This discrepancy may result from the fact that the 6-hour incubation time used in their study was insufficient to trigger cell response. Our results showed a positive correlation between filamentation and TNF- $\alpha$ release $(\mathrm{p}=0.0119)$ for $C$. parapsilosis. Candida orthopsilosis strains induced TNF- $\alpha$ levels similar to the clinical isolates, whereas C. metapsilosis isolates induced the production of lower amounts, which is in agreement with Gácser et al. [19] who showed that C. metapsilosis appears as the less virulent of the three species of the $C$. parapsilosis complex. Nevertheless, recent literature indicates that $C$. metapsilosis can be retrospectively identified at a frequency similar to C. orthopsilosis and from virtually all body sites $[37,38]$. In addition, a metagenomic study has found $C$. metapsilosis sequences in the oral cavity of healthy carriers, suggesting the possibility of oral commensalism for this species [39].

\section{Conclusions}

Overall, this report evidences for the first time that environmental and clinical C. parapsilosis isolates behave differently in contact with macrophages, 
indicating that environmental strains cause a higher cellular damage and seem to be more prone to resist to macrophage killing. Since nosocomial fungal infections progress rapidly, and C. parapsilosis is frequently isolated from the hospital settings, there is a critical need for more efforts toward prevention, early diagnosis, and effective treatment of these infections. Among the preventive measures the environmental surveillance and strict application of cleaning procedures are of major importance to prevent the onset of hospital outbreaks.

\section{Methods}

\section{Candida isolates and preparation of cell suspensions}

Forty-five C. parapsilosis isolates, eight C. orthopsilosis isolates, and four C. metapsilosis isolates were used in this study (Table 1). Twenty-five of the C. parapsilosis isolates were from bloodstream infections, and 20 were obtained from the hospital environment, including bedside tables, doors knobs, surfaces, and air. The identity of the isolates was confirmed at the species level by locus specific amplification [40] or by sequencing the ribosomal ITS region [41]. Yeast cells were grown overnight at $37^{\circ} \mathrm{C}$ in YEPD medium ( $2 \%$ glucose, $1 \%$ bacto peptone, and $2 \%$ yeast extract), recovered by centrifugation, washed in sterile PBS buffer, and a suspension of $2 \times 10^{7}$ cells $/ \mathrm{ml}$ was prepared in Dulbecco's Modified Eagle's Medium (DMEM).

\section{Macrophage culture and determination of candidacidal activity}

The murine macrophage-like cell line J774A.1 (American Type Culture Center number TIB 67Ralph and Nakoinz, 1975) was cultured in complete DMEM supplemented with $10 \%$ heat-inactivated fetal calf serum (FBS), at $37^{\circ} \mathrm{C}$ in a $5 \% \mathrm{CO}_{2}$ atmosphere. After confluent growth, macrophage cells were recovered, washed, and re-suspended in DMEM to a final concentration of $4 \times$ $10^{5} \mathrm{cells} / \mathrm{ml}$. Yeast killing was assessed by using a multiplicity of infection (MOI) of 1:10 in 24 well tissue-culture plates (Orange) for 60 minutes, at $37^{\circ} \mathrm{C}$ in a $5 \%$ $\mathrm{CO}_{2}$ atmosphere. After incubation macrophage cells were lysed with $800 \mu \mathrm{l}$ of cold water and wells scrapped to ensure removal of all the yeast cells. Lysates were serially diluted and plated on YEPD agar to determine the percentage of viable yeast cells. Controls consisted of yeast cells grown in the same conditions but without macrophages. Candidacidal activity (\%) was calculated using the following formula: [(CFU of control well CFU of test well)/CFU of control well] $\times 100$. Each strain was tested in triplicate.

\section{Analysis of C. parapsilosis morphology during macrophage infection}

Yeast cell morphology in contact with macrophages was evaluated by co-incubating the macrophage cell line with Candida cells, as described above. Macrophage cells were seeded into 24 well tissue-culture plates containing a plastic coverslip in each well (Nunc, Rochester, USA) to allow macrophage adherence. After 1, 4, 8, 10 and 12 hours of co-incubation coverslips were removed, fixed with $10 \%$ formol on ethanol, and Hemacolor (Merck, New Jersey, EUA) stained. Different fields were analyzed under a Leica DM5000B light microscope and images captured with a Leica DFC350FX camera.

\section{Macrophage death assessment}

Kinetic of macrophage death was assessed by incubating macrophages with $C$. parapsilosis at a MOI of 1:10 as previously described. Macrophage death was assayed by determining the percentage of cells with plasma membranes permeable to propidium iodide (PI) after 1, 2, 3, $4,6,8,10$ and 12 hours of co-incubation. Cells on the coverslips were stained with $1 \mu \mathrm{g} / \mathrm{ml} \mathrm{PI}$ at room temperature for $10 \mathrm{~min}$ in the dark, and observed using a Leica DM5000B fluorescence microscope. At each time point, images were taken and approximately 1000 cells were counted in independent fields. The percentage of macrophage cells permeable to PI was calculated as described by Shin et al. [24].

\section{Lactate dehydrogenase (LDH) measurement}

The release of $\mathrm{LDH}$ from cells into the medium was monitored as a measure of cell damage. LDH released in the medium from macrophage cultures (negative control) and from macrophages co-incubated with C. parapsilosis, C. orthopsilosis and C. metapsilosis was measured after $12 \mathrm{~h}$ incubation by using the Cytotoxicity Detection Kit PLUS (LDH) (Roche Diagnostics Corporation, Indianapolis, USA), according to the manufacturer's instructions.

\section{Cytokine measurement}

TNF- $\alpha$ production by macrophages infected with the strains in study was measured using the Mouse TNF $\alpha$ ELISA ReadySETGoKit (eBioscience, San Diego, CA, USA), according to the manufacturer's instructions.

\section{Secreted aspartic proteinase and phospholipase production}

The production of secreted aspartic proteinases (Sap) and phospholipases by isolates of C. parapsilosis, $C$. orthopsilosis and C. metapsilosis was determined as previously described [42]. One C. albicans producer strain (SC5314) was added as a positive control.

\section{Filamentation assay}

Filamentation was assessed by seeding $200 \mu \mathrm{l}$ of the prepared cell suspensions into 24 well tissue-culture plates (Orange), and incubating at $37^{\circ} \mathrm{C}$ in a $5 \% \mathrm{CO} 2$ 
atmosphere for 12 hours. An aliquot of each suspension was then smeared onto a glass slide and images were taken with a Leica DM5000B light microscope.

\section{Statistical analysis}

Unless otherwise stated, results shown are the mean of three independent experiments \pm SD. Statistical significance of results was determined by the $\mathrm{T}$ student test or the $\chi^{2}$-test. Results were considered statistically significant when two-tailed $\mathrm{p}$ values were less than 0.05 . All calculations were performed with GraphPad Prism 5 software.

\section{Acknowledgements}

This research was supported by FEDER funds through the Operational Programme COMPETE and national funds through Fundação para a Ciência e Tecnologia (FCT), in the scope of project PEst-C/BIA/U/4050/2011. Raquel Sabino received a fellowship from FCT (contract BD/22100/2005). We are indebted to Frédéric Grenouillet, Guillermo Garcia-Effron, Zélia Videira and Maria da Luz Martins for kindly providing part of the isolates used in this study.

\section{Author details}

'CBMA - Centre of Molecular and Environmental Biology, Department of Biology, University of Minho, Braga, Portugal. '2Laboratório de Micologia, Departamento de Doenças Infecciosas, Instituto Nacional de Saúde Dr. Ricardo Jorge, Lisboa, Portugal.

\section{Authors' contributions}

PS and CP conceived and designed the study. RS, PS, and CC performed the experiments; RS, PS, LR, and CP analyzed the data; RS, PS and CP wrote the manuscript. All authors have read and approved the final version of the manuscript

Received: 18 February 2011 Accepted: 8 August 2011

Published: 8 August 2011

\section{References}

1. Moran GP, Sullivan DJ, Coleman DC: Emergence of non Candida albicans Candida species as pathogens. In Candida and Candidiasis. Edited by: Calderone RA. Washington DC: ASM Press; 2002:341-348.

2. Almirante B, Rodriguez D, Cuenca-Estrella M, Almela M, Sanchez F, Ayats J, Alonso-Tarres C, Rodriguez-Tudela JL, Pahissa A, the Barcelona Candidemia Project Study Group: Epidemiology, risk factors and prognosis of Candida parapsilosis bloodstream infections: case-control population-based surveillance study of patients in Barcelona, Spain, from 2002 to 2003. J Clin Microbiol 2006, 44:1681-1685.

3. Costa-de-Oliveira S, Pina-Vaz C, Mendonça D, Rodrigues AG: A first Portuguese epidemiological survey of fungaemia in a university hospital. Eur J Clin Microbiol Infect Dis 2008, 27:365-374.

4. Trofa D, Gácser A, Nosanchuk JD: Candida parapsilosis, an emerging fungal pathogen. Clin Microbiol Rev 2008, 21:606-625.

5. van Asbeck EC, Clemons KV, Stevens DA: Candida parapsilosis: a review of its epidemiology, pathogenesis, clinical aspects, typing, and antimicrobial susceptibility. Crit Rev Microbiol 2009, 35:283-309.

6. Sabino R, Veríssimo C, Brandão J, Alves C, Parada H, Rosado L, Paixão E, Videira Z, Tendeiro T, Sampaio P, Pais C: Epidemiology of candidemia in oncology patients: a 6-year survey in a Portuguese central hospital. Med Mycol 2010, 48:346-54.

7. Saiman L, Ludington E, Pfaller M, Rangel-Frausto S, Wiblin RT, Dawson J, Blumberg HM, Patterson JE, Rinaldi M, Edwards JE, Wenzel RP, Jarvis W: Risk factors for candidemia in neonatal intensive care unit patients. The National Epidemiology of Mycosis Survey Study Group. Pediatr Infect Dis J 2000, 19:319-24

8. Karlowicz MG, Rowen JL, Barnes-Eley ML, Burke BL, Lawson ML, Bendel CM, Shattuck KE, Horgan M, Albritton WL: The role of birth weight and gestational age in distinguishing extremely low birth weight infants at high risk of developing candidemia from infants at low risk: a multicenter study. Pediatr Res 2002, 51:301A.

9. Clerihew L, Lamagni TL, Brocklehurst P, McGuire W: Candida parapsilosis infection in very low birthweight infants. Arch Dis Child Fetal Neonatal Ed 2007, 92:F127-F129.

10. Muňoz P, Burillo A, Pouza E: Environmental surveillance and other control measures in the prevention of nosocomial fungal infections. Clin Microbiol Infect 2001, 7:38-45.

11. Sautour M, Dalle F, Olivieri C, L'ollivier C, Enderlin E, Salome E, Chovelon I, Vagner O, Sixt N, Fricker-Pap V, Aho S, Fontaneau O, Cachia C, Bonnin A: A prospective survey of air and surface fungal contamination in a medical mycology laboratory at a tertiary care university hospital. Am J Infect Control 2009, 37:189-194.

12. Barchesi F, Caggiano G, Falconi Di Francesco L, Montagna MT, Barbuti S, Scalise G: Outbreak of fungemia due to Candida parapsilosis in a pediatric oncologic unit. Diagn microbial Infect Dis 2004, 49:269-271.

13. van Asbeck EC, Huang Y-C, Markham AN, Clemons KV, Stevens DA: Candida parapsilosis fungemia in neonates: genotyping results suggest healthcare workers hands as source, and review of published studies. Mycophatologia 2007, 164:287-293.

14. Hube B, Stehr F, Bossenz M, Mazur A, Kretschmar M, Schäfer W: Secreted lipases of Candida albicans: cloning, characterisation and expression analysis of a new gene family with at least ten members. Arch Microbiol 2000, 174:362-374

15. Khun DM, Mikherjee PK, Clark TA, Pujol C, Chandra J, Hajjeh RA, Warnock DW, Soil DR, Ghannoum MA: Candida parapsilosis characterization in an outbreak setting. Emerg Infect Dis 2004, 10:1074-1081.

16. Bramono K, amazaki M, Tsuboi R, Ogawa H: Comparison of proteinase, lipase and alpha-glucosidase activities from the clinical isolates of Candida species. Jpn J Infec Dis 2006, 59:73-76.

17. Owaki T, Meneshian A, Maemura K, Takao S, Wang D, Fuh KC, Bulkley GB, Klein AS: Endothelial cells potentiate phagocytic killing by macrophages via platelet-activating factor release. Am J Physiol Heart Circ Physiol 2000, 278: $\mathrm{H} 269-\mathrm{H} 276$.

18. Gácser A, Trofa D, Schäfer W, Nosanchuk JD: Targeted gene deletion in Candida parapsilosis demonstrates the role of secreted lipase in virulence. J Clin Invest 2007, 117:3049-3058.

19. Gácser A, Schafer W, Nosanchuk JS, Salomon S, Nosanchuk JD: Virulence of Candida parapsilosis, Candida orthopsilosis, and Candida metapsilosis in reconstituted human tissue models. Fungal Genet Biol 2007, 44:1336-1341.

20. Maródi L, Schreiber S, Anderson DC, MacDermott RP, Korchak HM Johnston RB Jr: Enhancement of macrophage candidacidal activity by interferon-y - increased phagocytosis, killing, and calcium signal mediated by a decreased number of mannose receptors. $J$ Clin Invest 1993, 91:2596-2601.

21. Camargo MR, Venturini J, Vilani-Moreno FR, Arruda MSP: Modulation of macrophage cytokine profiles during solid tumor progression: susceptibility to Candida albicans infection. BMC Infectious Diseases 2009, 9:98-106.

22. Lorenz MC, Fink GR: Life and death in a macrophage: role of the glyoxylate cycle in virulence. Eukaryot Cell 2002, 1:657-662.

23. Orsi CF, Colombari B, Blasi E: Candida metapsilosis as the least virulent member of the C. parapsilosis complex. Med Mycol 2010, 48:1024-1033.

24. Shin YK, Kim KY, Paik YK: Alterations of protein expression in macrophages in response to Candida albicans infection. Mol Cells 2005, 20:271-279.

25. Tavanti A, Campa D, Bertozzi A, Pardini G, Naglik JR, Barale R, Senesi S: Candida albicans isolates with different genomic backgrounds display a differential response to macrophage infection. Microbes Infect 2006, 8:791-800

26. Stehr F, Gácser A, Kretschmar M, Mähnss B, Neuber K, Hube B, Schäfer W: Expression analysis of the Candida albicans lipase gene family during experimental infections and in patient samples. FEMS Yeast Res 2004 4:401-408.

27. Schaller M, Borelli C, Korting HC, Hube B: Hydrolytic enzymes as virulence factors of Candida albicans. Mycoses 2005, 48:365-377.

28. De Bernardis F, Mondello F, San Millán R, Pontón J, Cassone A: Biotyping and virulence properties of skin isolates of Candida parapsilosis. J Clin Microbiol 1999, 37:3481-3486 
29. Pichová I, Pavlicková L, Dostál J, Dolejsí E, Hrusková-Heidingsfeldová O Weber J, Ruml T, Soucek M: Secreted aspartic proteases of Candida albicans, Candida tropicalis, Candida parapsilosis and Candida lusitaniae. Eur J Biochem 2001, 268:2669-2677.

30. Khun D, Chandra J, Mukherjee PK, Ghannoum MA: Comparison of biofilms formed by Candida albicans and Candida parapsilosis on bioprosthetic surfaces. Infect Immun 2002, 70:878-888.

31. Ozkan S, Kaynak F, Kalkanci A, Abbasoglu U, Kustimur S: Slime production and proteinase activity of Candida species isolated from blood samples and the comparison of these activities with minimum inhibitory concentration values of antifungal agents. Mem Inst Oswaldo Cruz 2005, 100:319-324.

32. Dagdeviren M, Cerikcioglu N, Karavus M: Acid proteinase, phospholipase, and adherence properties of Candida parapsilosis strains isolated fron clinical specimens of hospitalized patients. Mycoses 2005, 48:321-326.

33. Lin DM, Wu LC, Rinaldi MG, Lehmann PF: Three distinct genotypes within Candida parapsilosis from clinical sources. J Clin Microbiol 1995, 33:1815-1821

34. Levitz SM: Interactions of Toll-like receptors with fungi. Microb Infect 2004, 6:1351-1355

35. Romani L: Immunity to fungal infections. Nat Rev Immunol 2004, 4:11-24.

36. Zelante T, Montagnoli C, Bozza S, Gaziano R, Bellocchio S, Bonifazi P, Moretti S, Fallarino F, Puccetti P, Romani L: Receptors and pathways in innate antifungal immunity: the implication for tolerance and immunity to fungi. Adv Exp Med Biol 2007, 590:209-221.

37. Kocsubé S, Tóth M, Vágvölgyi C, Dóczi I, Pesti M, Pócsi I, Szabó J, Varga J: Occurrence and genetic variability of Candida parapsilosis sensu lato in Hungary. J Med Microbiol 2007, 56(Pt 2):190-5.

38. Hensgens LA, Tavanti A, Mogavero S, Ghelardi E, Senesi S: AFLP genotyping of Candida metapsilosis clinical isolates: Evidence for recombination. Fungal Genet Biol 2009, 46(10):750-758.

39. Ghannoum MA, Jurevic RJ, Mukherjee PK, Cui F, Sikaroodi M, Naqvi A, Gillevet PM: Characterization of the Oral Fungal Microbiome (Mycobiome) in Healthy Individuals. PLoS Pathog 2010, 6(1):e1000713.

40. Sabino R, Sampaio P, Rosado L, Stevens DA, Clemons KV, Pais C: New polymorphic microsatellite markers able to distinguish among. Candida parapsilosis senso stricto isolates. J Clin Microbiol 2010, 48:1677-1682.

41. Sampaio JP, Gadanho M, Santos M, Duarte FL, Pais C, Fonseca A, Fell JW: Polyphasic taxonomy of the basidiomycetous yeast genus Rhodosporidium: Rhodosporidium kratochvilovae and related anamorphic species. Int J Syst Evol Microbiol 2001, 51:687-697.

42. Al-Abeid H, Abu-Elteen $\mathrm{K}$, Elkarmi A, Hamad M: Isolation and characterization of Candida spp. in Jordanian cancer patients: prevalence, pathogenic determinants and antifungal sensitivity. Jpn J Infect Dis 2004, 57:279-284.

doi:10.1186/1471-2180-11-180

Cite this article as: Sabino et al:: Isolates from hospital environments are the most virulent of the Candida parapsilosis complex. BMC Microbiology 2011 11:180

\section{Submit your next manuscript to BioMed Central and take full advantage of:}

- Convenient online submission

- Thorough peer review

- No space constraints or color figure charges

- Immediate publication on acceptance

- Inclusion in PubMed, CAS, Scopus and Google Scholar

- Research which is freely available for redistribution 